\title{
Pieri rule for the affine flag variety
}

\author{
Seung Jin Lee $\|^{\dagger}$ \\ School of Mathematics, Korea Institute for Advanced Study, 85 Hoegiro Dongdaemun-gu, Seoul 130-722, Republic \\ of Korea.
}

\begin{abstract}
We prove the affine Pieri rule for the cohomology of the affine flag variety conjectured by Lam, Lapointe, Morse and Shimozono. We study the cap operator on the affine nilHecke ring that is motivated by Kostant and Kumar's work on the equivariant cohomology of the affine flag variety. We show that the cap operators for Pieri elements are the same as Pieri operators defined by Berg, Saliola and Serrano. This establishes the affine Pieri rule.

Résumé. Nous prouvons la règle affine Pieri pour la cohomologie de la variété affine de drapeau conjecturé par Lam, Lapointe, Morse et Shimozono. Nous étudions l'opérateur de bouchon sur l'affine nilHecke anneau qui est motivé par le travail de Kostant et Kumar sur la cohomologie équivariante de la variété affine de drapeau. Nous montrons que les opérateurs de capitalisation pour les éléments Pieri sont les mêmes que les opérateurs Pieri définies par Berg, Saliola et Serrano. Ceci établit la règle affine Pieri.
\end{abstract}

Keywords: affine flag variety, $k$-Schur function, nilCoxeter algebra, Pieri rule, strong Schur function.

\section{Introduction}

Affine Schubert calculus is a subject that ties combinatorics, algebraic geometry and representation theory together. Its modern development is motivated by the relation between $k$-Schur functions and the (co)homology of the affine Grassmannian of $S L(n) . k$-Schur functions were introduced by Lapointe, Lascoux, Morse in [LLM03] in the study of Macdonald polynomial positivity, a mostly combinatorial branch of symmetric function theory.

Peterson [Pet97] identified the equivariant homology of the affine Grassmannian with a subalgebra of the affine nilHecke algebra $\mathbb{A}$, now called the Peterson algebra. Lam proved that $k$-Schur functions can be identified with the Schubert classes of the homology of the affine Grassmannian of $S L(n)$ in [Lam08] . The nilHecke ring acts as divided difference operators on the equivariant cohomology of Kac-Moody partial flag varieties. By using the correspondence, one can investigate problems about the (co)homology of the affine Grassmannian of $S L(n)$ by translating them into the theory of $k$-Schur functions and understanding the combinatorics of $k$-Schur functions. There are affine analogues of the classical theory of Pieri rules, tableaux, and Stanley symmetric functions. See [Lam06, ML05, ML07].

\footnotetext{
${ }^{\dagger}$ Email: lsjin@kias.re.kr.

1365-8050 (c) 2015 Discrete Mathematics and Theoretical Computer Science (DMTCS), Nancy, France
} 
Lam, Lapointe, Morse and Shimozono introduced the strong Schur functions, indexed by elements in the affine symmetric group $W_{\text {af }}$ in [LLMS10]. These strong Schur functions generalize the $k$-Schur functions combinatorially. They conjectured a series of properties of strong Schur functions such as the symmetry of the strong Schur functions. Berg, Saliola and Serrano studied the Pieri operators acting on the affine nilCoxeter ring $\mathbb{A}_{0}$ in [BSS13, BSS14] to establish some of conjectures in [LLMS10].

In this extended abstract, we describe ideas for proving the affine Pieri rule for the cohomology of the affine flag variety conjectured in [LLMS10]. We introduce the cap operators acting on the affine nilCoxeter ring $\mathbb{A}_{0}$ by investigating the work in [KK86] and show that the cap operators for Pieri elements are the same as the Pieri operators defined in [BSS14] by using the strong strips. The affine Pieri rule gives us geometric interpretation of the skew strong Schur functions as an affine Grassmannian part of the cap product of the Schubert classes in (co)homology of the affine flag variety. We now describe these two operators.

\subsection{Cap operators}

Let us consider the affine type $A$ root datum and corresponding Kac-Moody flag variety $\widetilde{F l}$ (See [KK86] for details). Let $\left\{\xi^{w} \mid w \in W_{\text {af }}\right\}$ denote the Schubert basis for the equivariant cohomology $H_{T}^{*}(\widetilde{F l})$ of $\widetilde{F l}$. One of main problems in affine Schubert calculus is to find a combinatorial formula for the structure constants $p_{u, v}^{w}$ where $\xi^{u} \xi^{v}=\sum_{w} p_{u, v}^{w} \xi^{w}$. In [KK86], Kumar and Kostant identified the torus-equivariant cohomology $H_{T}^{*}(\widetilde{F l})$ of the affine flag variety and the dual of the nilHecke ring. Using this connection, one can compute the structure constants $p_{u, v}^{w}$ by calculating the coproduct structure constants of $\mathbb{A}$. More precisely, we have

$$
\Delta\left(A_{w}\right)=\sum_{u, v \in W_{\mathrm{af}}} p_{u, v}^{w} A_{u} \otimes A_{v} .
$$

For $u \in W_{\text {af }}$, a cap operator $D_{u}$ on $\mathbb{A}_{0}$ is defined by

$$
D_{u}\left(A_{w}\right)=\sum_{\substack{v \in W_{\text {af }} \\ \ell(v)=\ell(w)-\ell(u)}} p_{u, v}^{w} A_{v} .
$$

Geometrically, the cap operator is the cap product on the ordinary homology and cohomology of the affine flag variety. More precisely, the cap operator $D_{u}$ can be considered as an element in $H^{*}(\widetilde{F l})$ and $A_{w}$ can be considered in $H_{*}(\widetilde{F l})$ so that the cap product $H^{*}(\widetilde{F l}) \times H_{*}(\widetilde{F l}) \rightarrow H_{*}(\widetilde{F l})$ can be described by $\left(D_{u}, A_{w}\right)=D_{u}\left(A_{w}\right)$. Note that the cap operator only keep track of ordinary cohomology since $p_{u, v}^{w}$ is constant when $\ell(v)=\ell(w)-\ell(u)$.

Let $\rho_{i}$ be the Pieri element $s_{i-1} \ldots s_{1} s_{0}$ in $W_{\text {af }}$ where indices are taken modulo $n$. We study the cap operators $D_{\rho_{i}}$ for $\rho_{i}$ and show that $D_{\rho_{i}}$ satisfy the properties of the Pieri operators $D_{i}^{\prime}$ that Berg, Saliola and Serrano proved in [BSS14]. This establishes the following main theorems equivalent to the affine Pieri rule conjectured in [LLMS10].

Theorem 1.1 For $w, u \in W_{\text {af }}$ with $i=\ell(w)-\ell(u) \in \mathbb{N}, p_{\rho_{i}, u}^{w}$ counts the number of strong strips from $w$ to $u$. 
Theorem 1.2 Let $w \in W_{\text {af }}$ and $1 \leq m$. Then in $H^{*}(\widetilde{F l})$ we have

$$
\xi^{\rho_{m}} \xi^{w}=\sum_{S} \xi^{\text {outside }(S)}
$$

where the sum runs over strong strips $S$ of size $m$ such that inside $(S)=w$.

Note that Theorem 1.1 and 1.2 are equivalent. As a corollary, one can compute $p_{v, u}^{w}$ for $v=\rho_{m}^{-1}$ or $v=s_{i+m-1} s_{i+m-2} \cdots s_{i+1} s_{i}$ for any $i$ by applying automorphisms on Dynkin diagram of affine type $A$, namely, the map $j \mapsto-j$ and $j \mapsto j+i$ for $j \in \mathbb{Z} / n \mathbb{Z}$.

The paper is structured as follows. In section 2, we recall some notions about the affine symmetric group, $k$-Schur functions, strong Schur functions and root systems. In section 3, we define the affine nilHecke ring $\mathbb{A}$ and study its properties. In section 4 , we recall some statements concerning the affine flag variety and its equivariant cohomology as well as the relationship between the equivariant cohomology of affine flag variety and the coproduct structure of the affine nilHecke ring. In section 5, we define the cap operator. We show that these cap operators for Pieri elements agree with the Pieri operators defined by Berg, Saliola and Serrano. We also prove the affine Pieri rule for the ordinary cohomology of the affine flag variety. In section 6, we apply the affine Pieri rule to show that the structure constants $p_{u, v}^{w}$ for the cohomology of the affine flag variety can be described in terms of strong Schur functions when $u$ is 0 -Grassmannian. This also gives a geometric interpretation of the skew strong Schur functions.

\section{Preliminaries}

\subsection{Affine symmetric group}

Positive integers $n \geq 2$ and $k=n-1$ will be fixed throughout the extended abstract. Let $W_{\text {af }}$ denote the affine symmetric group with simple generators $s_{0}, s_{1}, \ldots, s_{n-1}$ satisfying the relations

$$
\begin{aligned}
s_{i}^{2} & =1 & \\
s_{i} s_{i+1} s_{i} & =s_{i+1} s_{i} s_{i+1} & \\
s_{i} s_{j} & =s_{j} s_{i} & \text { if } i-j \neq 1,-1 .
\end{aligned}
$$

where indices are taken modulo $n$. An element of the affine symmetric group may be written as a word in the generators $s_{i}$. A reduced word of the element is a word of minimal length. The length of $w$, denoted $\ell(w)$, is the number of generators in any reduced word of $w$.

The Bruhat order, also called strong order, on affine symmetric group elements is a partial order where $u<w$ if there is a reduced word for $u$ that is a subword of a reduced word for $w$. If $u<w$ and $\ell(u)=\ell(w)-1$, we write $u \lessdot w$. It is well-known that $u \lessdot w$ if and only if there exists a reflection $t \in\left\{w s_{i} w^{-1} \mid w \in W_{\mathrm{af}}, 0 \leq i<n\right\}$ such that $w=u t$ and $\ell(u)=\ell(w)-1$. For type $A$, the set $\left\{w s_{i} w^{-1} \mid w \in W_{\mathrm{af}}\right\}$ consists of transpositions $t_{i j}$. See [BB] for instance.

The subgroup of $W_{\text {af }}$ generated by $\left\{s_{1}, \cdots, s_{n-1}\right\}$ is naturally isomorphic to the symmetric group $W$. The 0-Grassmannian elements are minimal length coset representatives of $W_{\text {af }} / W$. 


\subsection{Symmetric functions}

Let $\Lambda$ denote the ring of symmetric functions. For a partition $\lambda$, we let $m_{\lambda}, h_{\lambda}, e_{\lambda}, s_{\lambda}$ denote the monomial, homogeneous, elementary and Schur symmetric functions, respectively, indexed by $\lambda$. Each of these families forms a basis of $\Lambda$.

Let $\Lambda_{(k)}$ denote the subalgebra generated by $h_{1}, h_{2}, \ldots, h_{k}$. The elements $h_{\lambda}$ with $\lambda_{1} \leq k$ form a basis of $\Lambda_{(k)}$. Let $\Lambda^{(k)}=\Lambda / I_{k}$ denote the quotient of $\Lambda$ by the ideal $I_{k}$ generated by $m_{\lambda}$ with $\lambda_{1}>k$. The image of elements $m_{\lambda}$ with $\lambda_{1} \leq k$ form a basis of $\Lambda^{(k)}$. Lam [Lam08, Theorem 7.1] showed that $\Lambda_{(k)}$ (resp. $\Lambda^{(k)}$ ) are isomorphic to homology(resp. cohomology) of affine Grassmannian as Hopf-algebras.

\section{$2.3 k$-Schur and Strong Schur functions}

There are many conjecturally equivalent definitions of $k$-Schur functions in [LLM $\left.{ }^{+} 14\right]$. We follow the definition of $k$-Schur functions in [LLMS10] using strong strips. Note that the marking of strong covers and strong strips defined in this extended abstract follows the notation of [BSS13]. This differs from the definition of strong covers and strips in [LLMS10] by reversing direction.

A marked strong cover $C=(u \stackrel{a}{\longrightarrow} v)$ consists of $u, v \in W_{\text {af }}$ and an integer $a$ such that $u=v t_{i j}, v \lessdot$ $u, a=u(j)=v(i)$ where $i \leq 0<j$. We use the notation inside $(C)=u$ and outside $(C)=v$.

A strong strip $\mathrm{S}$ of length $i$ from $u$ to $v$, denoted by $u \longrightarrow_{(i)} v$, is a path

$$
u \stackrel{a_{1}}{\longrightarrow} u_{1} \stackrel{a_{2}}{\longrightarrow} \cdots \stackrel{a_{i}}{\longrightarrow} u_{i}=v
$$

where $a_{1}>a_{2}>\ldots>a_{i}$. We let inside $(S)=u$, outside $(S)=v$, and $\operatorname{size}(S)=i$.

A strong tableau is a sequence $T=\left(S_{1}, S_{2}, \ldots\right)$ of strong strips $S_{i}$ such that outside $\left(S_{j}\right)=\operatorname{inside}\left(S_{j+1}\right)$ for all $j \in \mathbb{Z}_{>0}$ and $\operatorname{size}\left(S_{i}\right)=0$ for all sufficiently large $i$. We define inside $(T)=\operatorname{inside}\left(S_{1}\right)$ and $\operatorname{outside}(T)=\operatorname{outside}\left(S_{i}\right)$ for $i$ large. The weight $w t(T)$ of $T$ is the sequence

$$
w t(T)=\left(\operatorname{size}\left(S_{1}\right), \operatorname{size}\left(S_{2}\right), \ldots\right) .
$$

We say that $T$ has shape $u / v$ where $u=\operatorname{inside}(T)$ and $v=$ outside $(T)$ so that we have $u>v$.

Definition 2.1 For fixed $u, v \in W_{a f}$, define the Strong Schur function

$$
\operatorname{Strong}_{u / v}(x)=\sum_{T} x^{w t(T)}
$$

where T runs over the strong tableaux of shape $u / v$.

If $u$ is 0 -Grassmannian and $v$ is the identity element, $\operatorname{Strong}_{u}(x)$ is a $k$-Schur function $s_{\mathbf{c}(u)}^{(k)}$ where $\mathbf{c}$ is a bijection between the set of 0-Grassmannian elements and the set of $k$-bounded partitions. For details, see [LLMS10] for instance. 


\subsection{Root systems}

We shall assume basic familiarity with Weyl groups, root systems, and weights. See [Hum90] for details. Let $A=\left(a_{i j}\right)_{i, j \in I_{\mathrm{af}}}$ denote an affine Cartan matrix, where $I_{\mathrm{af}}=I \cup\{0\}$, so that $\left(a_{i j}\right)_{i, j \in I}$ is corresponding finite Cartan matrix. For type $\widetilde{A}_{n-1}$ (corresponding to $W_{\text {af }}$ ), we have $I_{\text {af }}=\mathbb{Z} / n \mathbb{Z}$ and

$$
a_{i j}=\left\{\begin{aligned}
2 & \text { if } i=j \\
-1 & \text { if }|i-j|=1 \\
0 & \text { otherwise. }
\end{aligned}\right.
$$

Let $R$ be the root system for $W$. Let $R^{+}, R^{-}$denote the set of positive roots, negative roots respectively. Let $\left\{\alpha_{i} \mid i \in I\right\}$ denote the simple roots and $\left\{\alpha_{i}^{\vee} \mid i \in I\right\}$ denote the simple coroots. Let $\theta$ denote the highest root $\alpha_{1}+\alpha_{2}+\ldots+\alpha_{n-1}$ of $R^{+}$.

Let $R_{\mathrm{af}}$ and $R_{\mathrm{af}}^{+}$denote the affine root system and positive affine roots. The positive simple affine roots (resp. coroots) are $\left\{\alpha_{i} \mid i \in I_{\text {af }}\right\}$ (resp. $\left\{\alpha_{i}^{\vee} \mid i \in I_{\text {af }}\right\}$ ). The null root $\delta$ is given by $\delta=\alpha_{0}+\theta=$ $\alpha_{0}+\cdots+\alpha_{n-1}$. Similarily, the canonical central element $c$ is given by $\alpha_{0}^{\vee}+\cdots+\alpha_{n-1}^{\vee}$. A root $\alpha$ is real if it is in $W_{\text {af }}$-orbit of the simple affine roots, and imaginary otherwise. The imaginary roots are exactly $\{k \delta \mid k \in \mathbb{Z} \backslash\{0\}\}$. Every real affine root is of the form $\alpha+k \delta$, where $\alpha \in R$ and $k \in \mathbb{Z}$. The root $\alpha+k \delta$ is positive if $k>0$, or if $k=0$ and $\alpha \in R^{+}$. Let $R_{\mathrm{re}}^{+}$denote the set of positive roots in $R_{\mathrm{af}}$.

Let $Q=\oplus_{i \in I} \mathbb{Z} \cdot \alpha_{i}$ denote the root lattice and let $Q^{\vee}=\oplus_{i \in I} \mathbb{Z} \cdot \alpha_{i}^{\vee}$ denote the coroot lattice. Let $P$ and $P^{\vee}$ be the weight lattice and coweight lattice respectively. We have inclusions $Q \subset P, Q^{\vee} \subset P^{\vee}$ and a map $Q_{\mathrm{af}}=\oplus_{i \in I_{\mathrm{af}}} \mathbb{Z} \cdot \alpha_{i} \rightarrow P$ given by sending $\delta$ to 0 . Let $\langle\cdot, \cdot\rangle$ denote the pairing between $P$ and $P^{\vee}$ satisfying $\left\langle\alpha_{i}^{\vee}, \alpha_{j}\right\rangle=a_{i j}$.

For a real root $\alpha$, we let $s_{\alpha}$ denote the corresponding reflection, defined by $s_{\alpha}=w s_{i} w^{-1}$ if $\alpha=w \cdot \alpha_{i}$. The reflection $s_{\alpha}$ acts on weights by $s_{\alpha} \lambda=\lambda-\langle\alpha, \lambda\rangle \alpha$. For a strong cover $v \lessdot w$, let $\alpha_{v, w}$ denote the unique positive root satisfying the equation $v^{-1} w=s_{a_{v, w}}$.

\section{NilHecke algebra}

\subsection{Definition}

Let $S=\operatorname{Sym}(P)$ be the polynomial ring having a variable for each free generator of $P$ and let $F=$ $\operatorname{Frac}(S)$ be the fraction field.

Define the $F$-vector space $F_{W_{\text {af }}}=\bigoplus_{w \in W_{\text {af }}} F w$ with basis $W_{\text {af }}$, with product given by

$$
(p v)(q w)=(p(v \cdot q))(v w) \quad \text { for } p, q \in F \text { and } v, w \in W_{\mathrm{af}} .
$$

For any real root $\alpha \in R_{\text {re }}$ define the element $A_{\alpha} \in F_{W_{\text {af }}}$ by

$$
A_{\alpha}=\alpha^{-1}\left(1-s_{\alpha}\right) \text {. }
$$

We write

$$
A_{i}=A_{\alpha_{i}} \quad \text { for } i \in I
$$


For $\alpha=w \cdot \alpha_{i} \in R_{\mathrm{re}}^{+}$, we have

$$
\begin{aligned}
w A_{i} w^{-1} & =A_{\alpha} \\
s_{\alpha} A_{\alpha} & =A_{\alpha} \\
A_{\alpha} s_{\alpha} & =-A_{\alpha} \\
A_{\alpha}^{2} & =0 .
\end{aligned}
$$

The $A_{i}$ satisfy the braid relations as the $s_{i}$ in $W_{\text {af }}$, i.e., $A_{i} A_{i+1} A_{i}=A_{i+1} A_{i} A_{i+1}$. Therefore it makes sense to define

$$
\begin{aligned}
& A_{w}=A_{i_{1}} \cdots A_{i_{l}} \quad \text { where } \\
& w=s_{i_{1}} \cdots s_{i_{l}} \quad \text { is a reduced decomposition. }
\end{aligned}
$$

One can check that

$$
A_{v} A_{w}= \begin{cases}A_{v w} & \text { if } \ell(v w)=\ell(v)+\ell(w) \\ 0 & \text { otherwise. }\end{cases}
$$

The nilCoxeter algebra $\mathbb{A}_{0}$ is the subring of $F_{W_{\text {af }}}$ generated by $A_{i}$ over $\mathbb{Z}$ for $i \in I_{\text {af }}$. The set $\left\{A_{w} \mid w \in\right.$ $\left.W_{\text {af }}\right\}$ forms a basis of $\mathbb{A}_{0}$ over $\mathbb{Z}$. The nilHecke algebra $\mathbb{A}$ is the subring of $F_{W_{\text {af }}}$ generated by $S$ and $\mathbb{A}_{0}$.

Remark 3.1 The affine nilHecke algebra $\mathbb{A}$ defined above is slightly different from those defined in [KK86]. Kostant and Kumar set $S=\operatorname{Sym}\left(P_{a f}\right)$ instead of $\operatorname{Sym}(P)$ where $P_{\text {af }}$ denote the affine weight lattice. We have a projection $P_{a f} \rightarrow P$ and the map induces $\operatorname{Sym}\left(P_{a f}\right) \rightarrow \operatorname{Sym}(P)$ which is compatible with Theorem 4.1. The image of the null root $\delta=\alpha_{0}+\theta$ in $P$ is 0 . Geometric interpretation of $P$ and $P_{a f}$ will be discussed in section 4

\subsection{Cyclically decreasing elements and Peterson algebra}

A word $s_{i_{1}} s_{i_{2}} \cdots s_{i_{l}}$ with indices in $\mathbb{Z} / n \mathbb{Z}$ is called cyclically decreasing if each letter occurs at most once and whenever $s_{i}$ and $s_{i+1}$ both occurs in the word, $s_{i+1}$ precedes $s_{i}$. For $J \varsubsetneqq \mathbb{Z} / n \mathbb{Z}$ there is the unique cyclically decreasing element $w_{J}$ with letters $\left\{s_{j} \mid j \in J\right\}$. For $i \in\{0,1, \ldots, n-1\}$, let

$$
\mathbf{h}_{i}=\sum_{\substack{J \subset I_{\mathrm{af}} \\|J|=i}} A_{w_{J}} \in \mathbb{A}_{0}
$$

where $\mathbf{h}_{0}=1$ and $\mathbf{h}_{i}=0$ for $i<0$ by convention. In [Lam06], Lam showed that the elements $\left\{\mathbf{h}_{i}\right\}_{i<n}$ commute and freely generate a subalgebra $\mathbb{B}$ of $\mathbb{A}_{0}$ called affine Fomin-Stanley algebra. It is well-known that $\mathbb{B}$ is isomorphic to $\Lambda_{(k)}$ via the map sending $\mathbf{h}_{i}$ to $h_{i}$. Therefore, the set $\left\{\mathbf{h}_{\lambda}=\mathbf{h}_{\lambda_{1}} \ldots \mathbf{h}_{\lambda_{l}} \mid \lambda_{1} \leq k\right\}$ forms a basis of $\mathbb{B}$.

\subsection{Coproduct structure of $\mathbb{A}$}

There is a coproduct structure on the nilHecke ring $\mathbb{A}$. Kumar and Kostant showed that the structure constants of the coproduct in $\mathbb{A}$ are the same as the structure constants of the equivariant cohomology ring of $\widetilde{F l}$. Details and proofs can be found in [KK86, LLM $^{+} 14$, Pet97].

Let $\Delta: F_{W_{\text {af }}} \rightarrow F_{W_{\text {af }}} \otimes_{F} F_{W_{\text {af }}}$ be the left $F$-linear map defined by

$$
\Delta(w)=w \otimes w \quad \text { for all } w \in W_{\mathrm{af}}
$$


Theorem 3.2 The map $\Delta: F_{W_{a f}} \rightarrow F_{W_{a f}} \otimes_{F} F_{W_{a f}}$ induces the unique left S-module homomorphism $\Delta: \mathbb{A} \rightarrow \mathbb{A} \otimes_{S} \mathbb{A}$ such that

$$
\begin{aligned}
\Delta\left(A_{i}\right) & =A_{i} \otimes 1+s_{i} \otimes A_{i} & & \\
& =1 \otimes A_{i}+A_{i} \otimes s_{i} & & \text { for all } i \in I \\
\Delta(a b) & =\Delta(a) \Delta(b) & & \text { for all } a, b \in \mathbb{A} .
\end{aligned}
$$

For $w, u_{1}, u_{2} \in W_{\mathrm{af}}$, the equivariant Schubert structure constants $p_{u_{1}, u_{2}}^{w} \in S$ are defined as the coefficients in the expansion $\Delta\left(A_{w}\right)=\sum p_{u_{1}, u_{2}}^{w} A_{u_{1}} \otimes A_{u_{2}}$. The followings are properties of $p_{u_{1}, u_{2}}^{w}$.

\section{Theorem 3.3}

1. $p_{u_{1}, u_{2}}^{w}=0$ unless $w \geq u_{1}$ and $w \geq u_{2}$.

2. $p_{u_{1}, u_{2}}^{w}$ is homogeneous of degree $\ell\left(u_{1}\right)+\ell\left(u_{2}\right)-\ell(w)$.

3. [Gra01 Kum02] $(-1)^{\ell\left(u_{1}\right)+\ell\left(u_{2}\right)-\ell(w)} p_{u_{1}, u_{2}}^{w} \in \mathbb{Z}_{\geq 0}\left[\alpha_{i} \mid i \in I_{a f}\right]$.

The last property in Theorem 3.3 is called Graham positivity. One can use Theorem 3.2 to compute $p_{u_{1}, u_{2}}^{w}$ explicitly.

Theorem 3.4 [KK86] Let $w=s_{i_{1}} s_{i_{2}} \ldots s_{i_{l}}$ and $l=\ell(w)$. Then

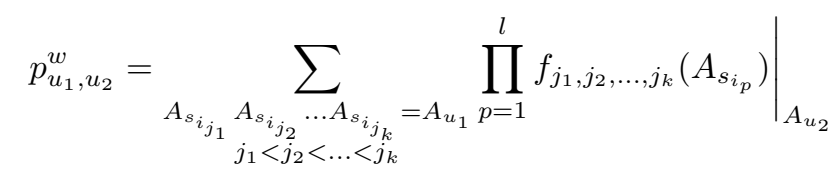

where $f_{j_{1}, j_{2}, \ldots, j_{k}}\left(A_{s_{i_{p}}}\right)=s_{i_{p}}$ if $p \in\left\{j_{1}, j_{2}, \ldots, j_{k}\right\}$ and $=A_{s_{i_{p}}}$ otherwise, and $\left.\sum c_{w} A_{w}\right|_{A_{u}}=c_{u}$.

\section{Affine flag varieties}

In this section, we define the Kac-Moody flag variety $\widetilde{F l}$ and establish the relationship between the equivariant cohomology of $\widetilde{F l}$ and the coproduct structure on $\mathbb{A}$. There are two definitions of Kac-Moody flag variety $\widetilde{F l}$ in [LLM $\left.{ }^{+} 14\right]$, but we only recall $\widetilde{F l}$ as the Kac-Moody flag ind-variety in [KK86, Kum02]. Let $G_{\text {af }}$ denote the Kac-Moody group of affine type associated with $S L(n)$ and let $B_{\text {af }}$ denote its Borel subgroup. The Kac-Moody flag ind-variety $\widetilde{F l}=G_{\text {af }} / B_{\text {af }}$ is paved by cells $B_{\text {af }} \dot{w} B_{\text {af }} / B_{\text {af }} \cong \mathbb{C}^{\ell(w)}$ whose closure $X_{w}$ is called the Schubert variety. Schubert variety defines a Schubert class $\left[X^{w}\right]_{T_{\mathrm{af}}} \in H_{T_{\mathrm{af}}}^{*}(\widetilde{F l})$ where $T_{\text {af }}$ is the maximal torus in $B_{\text {af }}$. For our setting, we consider $H_{T}^{*}(\widetilde{F l})$ where $T$ is a maximal torus in $S L(n)$. Kumar and Kostant [KK86] identified the equivariant cohomology ring of $\widetilde{F l}$ with the dual of nilHecke ring $\mathbb{A}$. By restricting the group action from $T_{\text {af }}$ to $T$, this identification shows that the structure constant of $H_{T}^{*}(\widetilde{F l})$ is the same as $p_{u, v}^{w}$ defined in Section 3.3 
Theorem 4.1 [KK86] The T-equivariant cohomology of $\widetilde{F l}$ has a basis $\left\{\left[X^{w}\right]_{T} \in H_{T}^{*}(\widetilde{F l})\right\}$ over $S \cong$ $H_{T}^{*}(p t)$. Moreover, the structure constants of $H_{T}^{*}(\widetilde{F l})$ are $p_{u_{1}, u_{2}}^{w}$, i.e.,

$$
\left[X^{u_{1}}\right]_{T}\left[X^{u_{2}}\right]_{T}=\sum p_{u_{1}, u_{2}}^{w}\left[X^{w}\right]_{T}
$$

Remark 4.2 The ordinary cohomology of $\widetilde{F l}$ has a basis $\left\{\left[X^{w}\right] \in H^{*}(\widetilde{F l})\right\}$ over $\mathbb{Z}$ and corresponding structure constants are just $\phi\left(p_{u_{1}, u_{2}}^{w}\right)$ where $\phi: S \rightarrow \mathbb{Z}$ is the evaluation at 0 .

\section{Pieri operators and Affine Pieri rule for type $A$}

Let $\phi: S \rightarrow \mathbb{Z}$ denote the map sending a polynomial to its constant term, called the evaluation map at 0 . It extends to the map $\phi: \mathbb{A} \rightarrow \mathbb{A}_{0}$ given by $\phi\left(\sum_{w} a_{w} A_{w}\right)=\phi\left(a_{w}\right) A_{w}$. Pieri elements $\rho_{i}$ are defined by $s_{i-1} s_{i-2} \ldots s_{1} s_{0}$ for $i>0$. For $w \in W_{\text {af }}$, let us define the cap operator $D_{w}$ on $\mathbb{A}$ (and $\mathbb{A}_{0}$ ) by

$$
D_{w}\left(A_{v}\right):=\phi\left(\sum_{u \in W_{\mathrm{af}}} p_{w, u}^{v} A_{u}\right)=\sum_{\substack{u \in W_{\mathrm{af}} \\ \ell(u)=\ell(v)-\ell(w)}} p_{w, u}^{v} A_{u} .
$$

If $w=\rho_{i}$, we call $D_{\rho_{i}}$ the Pieri operator and denote by $D_{i}$.

Note that $p_{w, u}^{v} \in \mathbb{Z}_{\geq 0}$ when $\ell(v)=\ell(w)+\ell(u)$ by Theorem 3.3. Finding a combinatorial formula for such $p_{w, u}^{v}$ is one of the important problems in Schubert calculus and it is not completely known even for finite flag varieties. In this section, we will describe main ideas showing that $D_{i}$ is the same as the Pieri operator $D_{i}^{\prime}$ defined by Berg, Saliola, Serrano in [BSS14]. This identification gives a combinatorial description of the Pieri rule for the ordinary cohomology of $\widetilde{F l}$ and therefore proves Theorem 1.1 Let us state properties of $D_{i}^{\prime}$ described in [BSS14].

Theorem 5.1 [BSS14 Theorem 4.8] Suppose $w \in W_{\text {af }}$ and $v \in W$. Then

$$
D_{i}^{\prime}\left(A_{w} A_{v}\right)=D_{i}^{\prime}\left(A_{w}\right) A_{v} .
$$

Lemma 5.2 [BSS14, Lemma 4.5] For $r<n$ and $i \geq 1$,

$$
D_{i}^{\prime}\left(\mathbf{h}_{r}\right)=\mathbf{h}_{r-i} .
$$

Theorem 5.3 [BSS14 Proposition 4.3] For $p<n, w \in W_{\text {af }}$ and $i \geq 1$, we have

$$
D_{i}^{\prime}\left(\mathbf{h}_{p} A_{w}\right)=\sum_{j=0}^{i} D_{j}^{\prime}\left(\mathbf{h}_{p}\right) D_{i-j}^{\prime}\left(A_{w}\right)=\sum_{j=0}^{i} \mathbf{h}_{p-j} D_{i-j}^{\prime}\left(A_{w}\right) .
$$

For $w \in W_{\text {af }}$, there is a unique decompostion $w=w_{\lambda} w(0)$ where $w_{\lambda}$ is 0 -Grassmannian and $w(0) \in$ $W$. Note that $\lambda=\mathbf{c}\left(w_{\lambda}\right)$ where $\mathbf{c}$ is the bijection between 0-Grassmannian elements and $k$-bounded partitions mentioned in section 2. In this setup, the set $\left\{\mathbf{h}_{\lambda} A_{w(0)}: w=w_{\lambda} w(0) \in W_{\mathrm{af}}\right\}$ forms a basis of $\mathbb{A}$ (See [BSS14]). This follows from the fact that $\mathbf{h}_{\lambda}$ has the unique 0-Grassmannian term $A_{w_{\lambda}}$. Therefore, above theorems uniquely determine $D_{i}^{\prime}$. It turns out that the same theorems hold for $D_{i}$. This implies that $D_{i}=D_{i}^{\prime}$ and Theorem 1.1 follows. 
Theorem 5.4 Suppose $w \in W_{\text {af }}$ and $v \in W$. Then

$$
D_{i}\left(A_{w} A_{v}\right)=D_{i}\left(A_{w}\right) A_{v}
$$

Lemma 5.5 [Lam08] Lemma 7.7] For $r<n$,

$$
\phi\left(\Delta\left(\mathbf{h}_{r}\right)\right)=\sum_{0 \leq j \leq r} \mathbf{h}_{r-j} \otimes \mathbf{h}_{j} .
$$

Theorem 5.6 For $r<n$ and all $i \geq 1$,

$$
D_{i}\left(\mathbf{h}_{r}\right)=\mathbf{h}_{r-i} .
$$

In order to prove analogue of Theorem 5.3 for $D_{i}$, we need the following lemmas.

Lemma 5.7 [Lam08] Lemma 7.2] Let $b \in \mathbb{B}$ and $s \in S$. Then

$$
\phi(b \cdot s)=\phi(s) b=b \cdot \phi(s)
$$

Lemma 5.8 Let $\psi: W_{a f} \rightarrow W_{\text {af }}$ be the group automorphism defined by $s_{i} \mapsto s_{i-1}$. The automorphism induces the ring automorphism $\psi: \mathbb{A}_{0} \rightarrow \mathbb{A}_{0}$. For $b \in \mathbb{B}$, we have

$$
\psi(b)=b .
$$

Theorem 5.9 For $p<n, w \in W_{a f}$ and $i \geq 1$, we have

$$
D_{i}\left(\mathbf{h}_{p} A_{w}\right)=\sum_{j=0}^{i} D_{j}\left(\mathbf{h}_{p}\right) D_{i-j}\left(A_{w}\right) .
$$

Theorems 5.4 5.6 and 5.9 imply that $D_{i}=D_{i}^{\prime}$, hence Theorem 1.1 .

\section{Applications}

Let $W^{0}$ denote the set of 0-Grassmannian elements in $W_{\text {af }}$. In this section, we describe how Theorem 1.1 implies the following conjecture, as stated in [LLMS10].

Theorem 6.1 [LLMS10. Conjecture 4.18] Let $w, u \in W_{\text {af }}$ be two affine permutations. Then

1. Strong ${ }_{w / u}(x) \in \Lambda$.

2. Strong $_{w / u}(x) \in \Lambda_{(n)}$.

3. $\operatorname{Strong}_{w / u}(x)=\sum_{v \in W^{0}} p_{u, v}^{w} \operatorname{Strong}_{v}(x)$.

Note that (1), (2) of Theorem 6.1 and $(3)$ when $w, v$ are in $W^{0}$ are proved in [BSS14]. To be more precise, they showed that for $w, v, u \in W^{0}, p_{u, v}^{w}$ is the coefficient of $\tilde{F}_{w}$ in $\tilde{F}_{u} \tilde{F}_{v}$, where $\tilde{F}_{w}$ is the affine Stanley symmetric function labeled by $w$. Lam, Lapointe, Morse and Shimozono introduced weak Schur functions in [LLMS10] defined by

$$
\operatorname{Weak}_{w / u}(x)=\sum_{\lambda}\left\langle\mathbf{h}_{\lambda} A_{u}, A_{w}\right\rangle_{\mathbb{A}_{0}} m_{\lambda}(x)
$$


for $w, u \in W_{\text {af }}$ where the summation is over all $k$-bounded partitions and $\langle\cdot, \cdot\rangle_{\mathbb{A}_{0}}$ is the inner product on $\mathbb{A}_{0}$ satisfying $\left\langle A_{w_{1}}, A_{w_{2}}\right\rangle_{\mathbb{A}_{0}}=\delta_{w_{1}, w_{2}}$. If $w \in W^{0}$ and $v$ is the identity element, Weak $w_{w / u}(x)$ is the affine Stanley symmetric function $\tilde{F}_{w}$.

Let $G r$ denote the affine Grassmannian associated with $S L(n, \mathbb{C})$. Since $G r \cong G_{\text {af }} / \mathcal{P}$ for the affine Kac-Moody group $G_{\text {af }}$ and its maximal parabolic subgroup $\mathcal{P}$, we have the Schubert bases

$$
\begin{aligned}
& \left\{\zeta^{w} \in H^{*}(G r, \mathbb{Z}) \mid w \in W^{0}\right\} \\
& \left\{\zeta_{w} \in H_{*}(G r, \mathbb{Z}) \mid w \in W^{0}\right\} .
\end{aligned}
$$

In [Lam08], Lam showed that $\zeta_{w}$ and $\zeta^{w}$ can be represented by $k$-Schur functions Strong $(x)$ and affine Stanley symmetric functions $\operatorname{Weak}_{w}(x)$ via the isomorphisms $H_{*}(G r) \cong \Lambda_{(n)}$ and $H^{*}(G r) \cong \Lambda^{(n)}$. In fact, affine insertion described in [LLMS10] offers a duality between weak and strong orders combinatorially.

Define the affine Cauchy kernel $\Omega_{n}(x, y)$ by

$$
\begin{aligned}
\Omega_{n}(x, y) & =\prod_{i}\left(1+y_{i} h_{1}(x)+y_{i}^{2} h_{2}(x)+\cdots+y_{i}^{n-1} h_{n-1}(x)\right) \\
& =\sum_{\lambda: \lambda_{1}<n} h_{\lambda}(x) m_{\lambda}(y) \\
& =\sum_{\lambda} m_{\lambda}(x) \tilde{h}_{\lambda}(y) .
\end{aligned}
$$

as an element in $\Lambda_{(n)}(x) \widehat{\otimes} \Lambda^{(n)}(y) \subset \Lambda(x) \widehat{\otimes} \Lambda^{(n)}(y)$ where $\tilde{h}_{\lambda}(y)$ is the image of $h_{\lambda}(y)$ in $\Lambda^{(n)}$. By the definition of $\Omega_{n}$ and Theorem 1.2 , we have

$$
\operatorname{Strong}_{w / u}(x)=\left\langle\Omega_{n} \cdot \xi^{u}, \xi^{w}\right\rangle_{\widetilde{F l}}
$$

where $\Omega_{n} \cdot \xi^{u} \in \Lambda \widehat{\otimes} H^{*}(G r)$ and $\langle\cdot, \cdot,\rangle_{\widetilde{F l}}$ is the inner product on $H^{*}(\widetilde{F l})$ satisfying $\left\langle\xi^{u}, \xi^{w}\right\rangle_{\widetilde{F l}}=\delta_{u, w}$. The duality between strong and weak orders produces the following affine Cauchy identity [LLMS10, Corollary 4.6].

Theorem 6.2 (Affine Cauchy Identity) The following identity holds in the formal power series ring:

$$
\Omega_{n}(x, y)=\sum_{w \in W^{0}} \operatorname{Strong}_{w}(x) \text { Weak }_{w}(y) \quad \text { in } \mathbb{Z}\left[\left[x_{1}, x_{2}, \ldots, y_{1}, y_{2}, \ldots\right]\right]
$$

Theorem 6.1 can be obtained by combining Theorem 6.2 and Equation (1) with slight modification.

Remark 6.3 The skew strong Schur function Strong ${ }_{w / u}(x)$ can be geometrically interpreted as the image of $D_{u}\left(\xi_{w}\right)$ under the projection $H_{*}(\widetilde{F l}) \rightarrow H_{*}(G r)$ where $D_{u}$ is the cap product acting on $H_{*}(\widetilde{F l})$. Indeed, by the definition of $D_{u}$ and Remark 4.2 we have $D_{u}\left(\xi_{w}\right)=\sum_{v} p_{u v}^{w} \xi_{v}$. Since the projection $H_{*}(\widetilde{F l}) \rightarrow H_{*}(G r)$ maps $\xi_{w}$ to Strong $g_{w}(x)$, it sends $D_{u}\left(\xi_{w}\right)$ to Strong ${ }_{w / u}(x)$ by Theorem 6.1 3$)$. Therefore, Strong $w_{w / u}(x)$ captures the 0-Grassmannian part of $D_{u}\left(\xi_{w}\right)$. Note that when $w$ is not 0Grassmannian, we have Strong $_{w}(x)=0$ (See [LLMS10]). 
Note that Lam, Lapointe, Morse and Shimozono defined the marked strong cover $w t_{i j} \rightarrow w$ where $i \leq l<j$ for a fixed constant $l$ instead of $i \leq 0<j$ in our setup. The affine Pieri rule conjectured in [LLMS10] is the following.

Theorem 6.4 [LLMS10 Conjecture 4.15] Let $w \in W_{\text {af }}$ and $1 \leq m$. Then in $H^{*}(\widetilde{F l})$ we have

$$
\xi^{\psi^{-l}\left(\rho_{m}\right)} \xi^{w}=\sum_{S} \xi^{\text {outside }(S)}
$$

where the sum runs over strong strips $S$ of size $m$ with respect to $l$ such that inside $(S)=w$.

We can obtain Theorem 6.4 by applying $\psi^{l}$ to Theorem 1.2 Recall that $\omega$ is the automorphism of $W_{\text {af }}$ sending $s_{i}$ to $s_{n-i}$. By applying $\omega$ to Theorem 1.2 , we get the dual affine Pieri rule.

Theorem 6.5 Let $w \in W_{\text {af }}$ and $1 \leq m$. Then in $H^{*}(\widetilde{F l})$ we have

$$
\xi^{\omega\left(\rho_{m}\right)} \xi^{w}=\sum_{S} \xi^{\omega(\text { outside }(S))},
$$

where the sum runs over strong strips $S$ of size $m$ such that inside $(S)=\omega(w)$.

Since the image of $\xi^{\omega\left(\rho_{m}\right)}$ under the projection $H^{*}(\widetilde{F l}) \rightarrow H^{*}(G r) \cong \Lambda^{(n)}$ is Weak $\left.\operatorname{Wo}_{\omega m}\right)=\tilde{e}_{m}$, the image of $e_{m} \in \Lambda^{(n)}$, so we recover the dual Pieri rule for the affine Grassmannian in [LLMS10].

\section{Acknowledgements}

I would like to thank Thomas Lam for helpful discussions.

\section{References}

[BB] A. Björner and F. Brenti. Combinatorics of Coxeter groups. Graduate Texts in Mathematics, 231:531-546.

[BSS13] C. Berg, F. Saliola, and L. Serrano. The down operator and expansions of near rectangular k-Schur functions. J. Combin. Theory Ser. A, 120(3):623-636, 2013.

[BSS14] C. Berg, F. Saliola, and L. Serrano. Pieri operators on the affine nilCoxeter algebra. Trans. Amer. Math. Soc., 366:531-546, 2014.

[FK99] S. Fomin and A. Kirillov. Quadratic algebras, Dunkl elements, and Schubert calculus. Adv. Geom., Progress in Mathematics, 172:147-182, 1999.

[Gra01] W. Graham. Positivity in equivariant Schubert calculus. Duke Math. J., 2001.

[Hum90] J. E. Humphreys. Reflection groups and Coxeter groups. Cambridge Studies in Advanced Mathematics, 29:xii+ 204 pp, 1990.

[Kas89] M. Kashiwara. The flag manifold of Kac-Moody Lie algebra. Algebraic Analysis, Geometry, and Number Theory, 1989. 
[KK86] B. Kostant and S. Kumar. The nil Hecke ring and cohomology of $G / P$ for a Kac-Moody group G. Adv. Math., 1986.

[KS09] M. Kashiwara and M. Shimozono. Equivariant K-theory of affine flag manifolds and affine Grothendieck polynomials. Duke Math. J., 2009.

[Kum02] S. Kumar. Kac-Moody groups, their flag varieties and representation theory. Progress in Mathematics, 2002.

[Lam06] T. Lam. Affine Stanley symmetric functions. Amer. J. Math., 2006.

[Lam08] T. Lam. Schubert polynomials for the affine Grassmannian. J. Amer. Math. Soc., 2008.

[LLM03] L. Lapointe, A. Lascoux, and J. Morse. Tableau atoms and a new Macdonald positivity conjecture. Duke Math. J., 2003.

$\left[\mathrm{LLM}^{+} 14\right]$ T. Lam, L. Lapointe, J. Morse, A. Schilling, M. Shimozono, and M. Zabrocki. k-Schur functions and affine Schubert calculus. Fields Institute Monographs, 2014.

[LLMS10] T. Lam, L. Lapointe, J. Morse, and M. Shimozono. Affine insertion and Pieri rules for the affine Grassmannian. Memoirs of the AMS, 2010.

[LSS10] T. Lam, A. Schilling, and M. Shimozono. Schubert polynomials for the affine Grassmannian of the symplectic group. Math. Z., 2010.

[ML05] J. Morse and L. Lapointe. Tableaux on $k+1$-cores, reduced words for affine permutations, and $k$-schur expansions. J. Combin. Theory Ser. A, 2005.

[ML07] J. Morse and L. Lapointe. A $k$-tableau characterization of $k$-Schur functions. Adv. Math., 2007.

[Pet97] D. Peterson. Lecture notes at MIT. 1997.

[Pon12] S. Pon. Affine Stanley symmetric functions for classical types. J. Combin. Theory Ser. A, 2012. 